\title{
Properties of short double-stranded RNAs carrying randomized base pairs: toward better controls for RNAi experiments
}

\author{
JULIAN A. ZAGALAK, ${ }^{1}$ MIRJAM MENZI, ${ }^{1}$ FABIAN SCHMICH, ${ }^{2}$ HARTMUT JAHNS, ${ }^{1}$ AFZAL M. DOGAR, ${ }^{1}$ \\ FLORIAN WULLSCHLEGER, ${ }^{1}$ HARRY TOWBIN, ${ }^{1}$ and JONATHAN HALL ${ }^{1}$ \\ ${ }^{1}$ Institute of Pharmaceutical Sciences, Department of Chemistry and Applied Biosciences, ETH Zurich, CH-8093 Zurich, Switzerland \\ ${ }^{2}$ Computational Biology Group (CBG), Department of Biosystems Science and Engineering, ETH Zurich, CH-4058 Basel, Switzerland
}

\begin{abstract}
Short interfering RNAs (siRNAs) are mediators of RNA interference (RNAi), a commonly used technique for selective downregulation of target gene expression. Using an equimolar mixture of $A, G, C$, and $U$ phosphoramidites during solid-phase synthesis, we introduced degenerate positions into RNA guide and passenger strands so that, when annealed, a large pool of distinct siRNA duplexes with randomized base pairs at defined sites was created. We assessed the randomization efficiency by deep sequencing one of the RNAs. All possible individual sequences were present in the pool with generally an excellent distribution of bases. Melting temperature analyses suggested that pools of randomized guide and passenger strands RNAs with up to eight degenerate positions annealed so that mismatched base-pairing was minimized. Transfections of randomized siRNAs (rnd-siRNAs) into cells led to inhibition of luciferase reporters by a miRNA-like mechanism when the seed regions of rnd-siRNA guide strands were devoid of degenerate positions. Furthermore, the mRNA levels of a select set of genes associated with siRNA off-target effects were measured and indicated that rnd-siRNAs with degenerate positions in the seed likely show typical non-sequence-specific effects, but not miRNA-like off-target effects. In the wake of recent reports showing the preponderance of miRNA-like off-target effects of siRNAs, our findings are of value for the design of a novel class of easily prepared and universally applicable negative siRNA controls.
\end{abstract}

Keywords: siRNA; degenerate base; randomized base; negative control; RNAi

\section{INTRODUCTION}

RNA-interference (RNAi) is a standard technique used for post-transcriptional gene silencing (Ghildiyal and Zamore 2009). It is mediated by short interfering RNAs (siRNAs) composed of a $\sim 19 \mathrm{bp}$, double-stranded (ds)-RNA with two nucleotide (nt) overhangs on the $3^{\prime}$ termini of both strands (Elbashir et al. 2001). After delivery into cells, the strand with the lower internal thermodynamic stability at its $5^{\prime}$ end is preferentially loaded into the RNA-induced silencing complex (RISC) (Khvorova et al. 2003; Schwarz et al. 2003). The remaining sense strand or "passenger" is cleaved by the RISC protein Argonaute2 (Ago2) (Matranga et al. 2005; Rand et al. 2005; Leuschner et al. 2006). The siRNA guide or antisense strand in the activated RISC binds to complementary target RNAs sequence-selectively and the target mRNA is cleaved opposite to the central positions of the siRNA guide by Ago2 (Elbashir et al. 2001). Although the guide strand is selected to be uniquely complementary to

Corresponding author: jonathan.hall@pharma.ethz.ch

Article published online ahead of print. Article and publication date are at http://www.rnajournal.org/cgi/doi/10.1261/rna.053637.115. the target mRNA, some highly homologous binding sites in other mRNAs are inevitably also suppressed by cross-hybridization, albeit less strongly, leading to sequence-dependent "off-target effects" (OTEs).

OTEs are considered a major nuisance in RNAi experiments that can lead to unexpected phenotypes and mislead researchers (Echeverri et al. 2006; Jackson et al. 2006; Jackson and Linsley 2010). In addition to Ago2-mediated sequence-dependent OTEs, additional sequence-dependent OTEs can derive from RISC activation of the passenger strand (Clark et al. 2008), immunostimulation (Sledz et al. 2003; Hornung et al. 2005; Judge et al. 2005), and RISC-mediated silencing of mRNAs in a microRNA (miRNA)-like fashion (Doench et al. 2003; Saxena et al. 2003; Birmingham et al. 2006; Jackson et al. 2006; Aleman et al. 2007; Vickers et al. 2009; Shin et al. 2010; Marine et al. 2012). Indeed, a recent

(C) 2015 Zagalak et al. This article is distributed exclusively by the RNA Society for the first 12 months after the full-issue publication date (see http://rnajournal.cshlp.org/site/misc/terms.xhtml). After 12 months, it is available under a Creative Commons License (Attribution-NonCommercial 4.0 International), as described at http://creativecommons.org/licenses/by$\mathrm{nc} / 4.0 /$. 
study declared that most of the phenotypic effects in three independent genome-wide siRNA screens stemmed from miRNA-like OTEs rather than from on-target silencing (Franceschini et al. 2014). Sequence-independent OTEs are also known and derive from saturation of RNAi machinery components (Jackson and Linsley 2010) and cell toxicity leading to cell death/growth inhibition. Different types of experimental controls have been introduced to help researchers validate the outcome of RNAi experiments (Whither RNAi? [Editorial] 2003; Echeverri et al. 2006). For instance, the redundancy approach uses independent effector siRNAs for a given target. Hence, a common phenotypic effect that is observed from use of multiple siRNAs can be attributed with reasonable certainty to target-mRNA silencing. More demanding are "rescue" experiments in which an siRNAinduced phenotype in cells is reverted by ectopic expression of a target gene bearing a mutated or deleted siRNA target site. Finally, a variety of negative control siRNAs are available: scrambled siRNAs where an effector siRNA sequence is shuffled to abolish target gene silencing; siRNAs that have minimal sequence complementarity to the transcriptome; or mismatched controls where a small number of mismatches (MMs) are introduced into the effector sequence and attenuate suppression of the target RNA (Buehler et al. 2012).

While exploring the properties of short RNAs containing randomized base pairs, we discovered that duplexes assembled from RNAs with randomized nucleotides at several selected positions hybridize to produce surprisingly thermodynamically stable duplexes that are substrates for RISC. SiRNAs with randomized base pairs (rnd-siRNAs) have attractive properties as a new class of pragmatic negative control siRNAs.

\section{RESULTS}

\section{Synthesis of RNAs containing randomized nucleotide positions}

As part of a program to study the multitargeting properties of short dsRNAs, we introduced randomized or degenerate positions into guide and passenger strands, so that when annealed a large combinatorial pool of distinct siRNA duplexes, with randomized matched and mismatched base pairs at defined sites, was created (Supplemental Table 1). For instance, with four degenerate positions, a library of 256 distinct RNA strands can theoretically be produced; with eight degenerate positions, 65,536 RNAs can be produced. After annealing of randomized strands, a huge combinatorial pool of individual siRNA duplexes can be produced, shaped by the thermodynamic stability of formed pairs. We postulated that the targeting profile of such libraries would be distributed across a very large number of individual targets and, because of the low concentrations of individual siRNAs, any sequence-dependent effects will likely be at biologically insignificant levels
(Buchholz et al. 2006; Myers et al. 2006; Parsons et al. 2009; Hannus et al. 2014). We synthesized 12 RNAs and assembled them into six randomized siRNAs (rnd-siRNAs) with four, six, or eight randomized positions (siRND1siRND6; Fig. 1A). For an efficient randomization of DNA oligonucleotides during synthesis, an equimolar mixture of the four deoxyribonucleotide phosphoramidites is often used (Zon et al. 1985; Mandecki 1990). We were not aware of any reports of variations in the coupling efficiencies of the four ribonucleotide phosphoramidites; therefore we used a 1:1:1:1 mixture of $A, G, C$, and $U$ phosphoramidites. To assess the efficiency of random ribonucleoside incorporation during solid-phase synthesis, we performed a deep-sequencing analysis of the guide strand of siRND3. The sequence coverage was well sufficient to cover all individual sequences in the library (see Materials and Methods). It also showed an excellent distribution of bases in the positions directly following G, A, and other randomized sites (Fig. 1B). On the other hand, reaction of a phosphoramidite mixture with cytidine, close to the $3^{\prime}$-terminus of the strand, showed a bias for incorporation of $G$, in the order $G>U>A>>C$. However, we cannot rule out that this skewing of the representation did not derive from an artifact during one of the steps of the library preparation for sequencing.

\section{SiRNAs with randomized base pairs adopt stable duplex structures}

Sense and antisense strands bearing randomized positions were annealed to form duplexes, which were analyzed using native polyacrylamide gel electrophoresis (PAGE) (Fig. 1C). The rnd-siRNAs migrated through the gel matrix more slowly than single-stranded controls and covered a comparable migratory path as a double-stranded commercially available siRNA control of undisclosed sequence (siCon), indicating their stable duplex structure. Subsequently, melting temperatures (Wada et al. 1980) of the rnd-siRNAs were calculated from melting curves obtained under standard conditions (Fig. 1D; Supplemental Fig. 1). Although we analyzed complex mixtures of siRNA duplexes, only single discrete inflection points were observed at a ramp rate of $0.1^{\circ} \mathrm{C} / \mathrm{min}$. The height of the first derivative of individual melting curves corresponded to the rate of transition; hence, broader melting curves (slower transitions) were observed for the population of siRNAs in siRNDs than for perfectly complementary siRNA controls. Only a minor degree of hysteresis was observed during the annealing and melting processes, suggesting that melting curves were obtained under thermodynamic equilibrium. The data revealed that, generally, increasing the number of degenerate positions in a duplex reduced the stability (lower $T_{\mathrm{M}} \mathrm{s}$ ) of an rnd-siRNA (Fig. 1D). Consistent with the results from the PAGE, the rndsiRNAs bearing up to eight randomized positions (siRND3, siRND6) were surprisingly stable. The $T_{\mathrm{M}}$ of siRND3 was compared with those of five perfectly complementary 
A

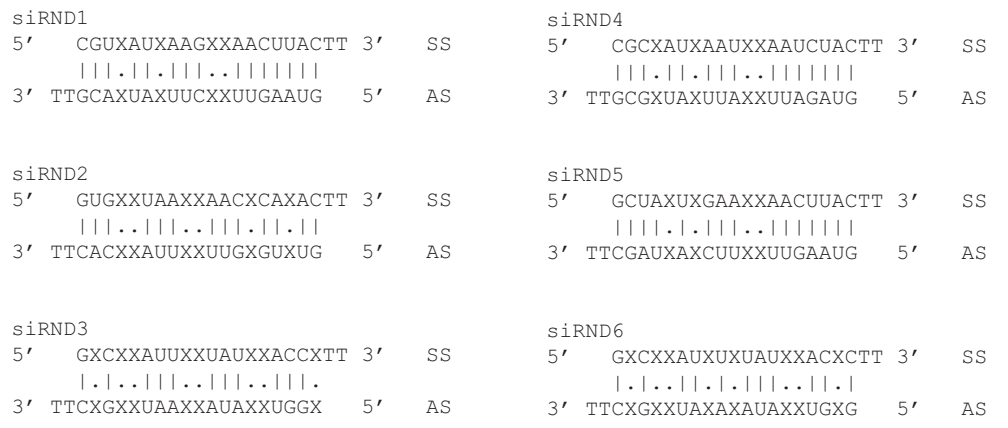

B

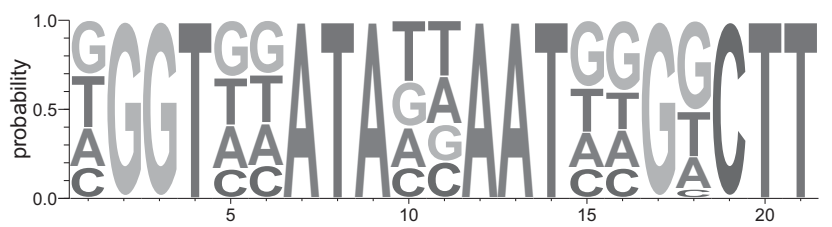

C

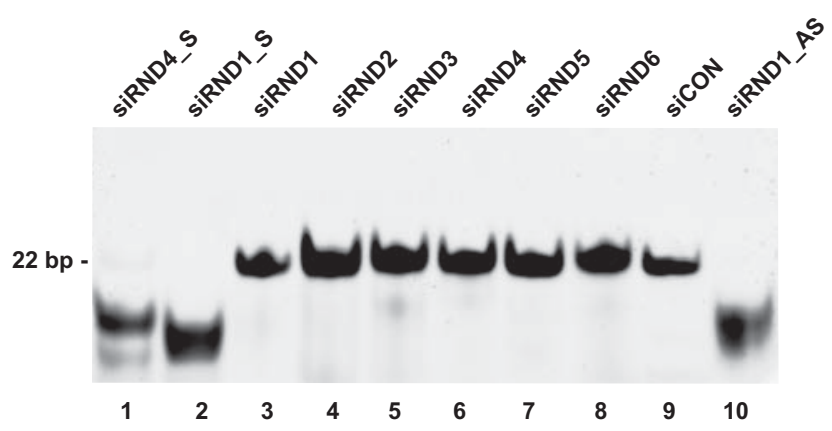

D

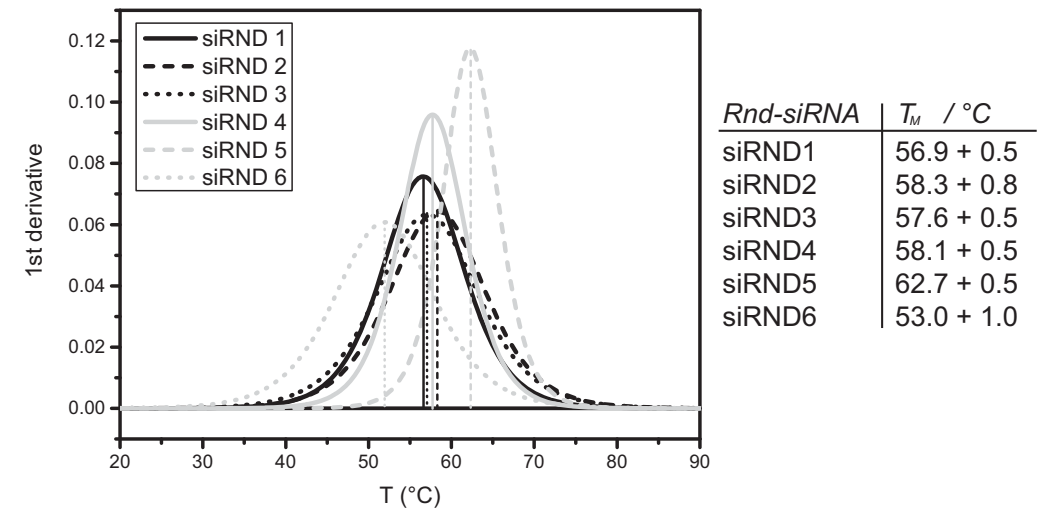

FIGURE 1. Synthesis and annealing properties of RNAs containing randomized nucleotide positions. (A) Sequences of the siRNDs 1-6. Watson-Crick base pairs are indicated with a dash. Randomized vicinal positions are indicated with a dot. (B) Deep-sequencing analysis of siRND3 antisense strand shows equal base distribution at nearly all randomized positions. Consensus sequence was created using WebLogo3. (C) Native polyacrylamide gel electrophoresis (PAGE) of siRNDs. Lanes 1,2, and 10 are loaded with single-stranded RNAs from siRND1 and siRND4 and serve as references; lane 9 contains siCon; lanes 3-8 contain siRNDs $1-6 .(D)$ Melting curve analysis of siRNDs. The graph shows the first derivative of melting curves. $T_{\mathrm{M}} \mathrm{s}$ listed in the table represent the mean of triplicate measurements \pm SD.

siRNA homologs that differed in the number of $\mathrm{G}-\mathrm{C}$ base pairs. The variant base pairs (either A-U or G-C) were positioned at identical locations as the randomized base pairs of siRND3 (Fig. 2A). As expected, we found that increasing $\mathrm{G}-\mathrm{C}$ pairs content in the siRNAs raised the $T_{\mathrm{M}}$ values from $54^{\circ} \mathrm{C}$
(siRNA3-0GC: $7 \mathrm{~A}-\mathrm{U}$ pairs) to $76^{\circ} \mathrm{C}$ (siRNA3-7GC: 7 G-C pairs) (Fig. 2B; Supplemental Fig. 1). Thus, siRND3 possessed a comparable thermal stability to that of a perfectly complementary siRNA homolog carrying one to two GC pairs (compare $T_{\mathrm{M}}$ of siRNA3-1GC). Intuitively, it seemed likely that the population would also comprise a significant fraction of mismatched duplexes (and G$\mathrm{U}$ pairs), not least in part because of the imperfect nucleoside distributions of the single strands (Fig. 1B). We could not envisage an experiment to determine the fraction of mismatched duplexes present in an rnd-siRNA. Instead, we selected one siRNA sequence (siRNA3) based on the scaffold of siRND3 and introduced a series (zero, two, four, or six) of mismatches (MMs) into the sense strand at identical locations as the randomized sites of siRND3 (Fig. 2C). The complementary duplex siRNA3 had a $T_{\mathrm{M}}$ of $70^{\circ} \mathrm{C}$; with two MMs it dropped to $58^{\circ} \mathrm{C}$; with four $\mathrm{MMs}$ it was $35^{\circ} \mathrm{C}$; and with six MMs it was $29^{\circ} \mathrm{C}$ (Fig. 2D; Supplemental Fig. 1). SiRND3 exhibited a $\Delta T_{\mathrm{M}}$ of $-12^{\circ} \mathrm{C}$ compared with its fully complementary counterpart, and a $\Delta T_{\mathrm{M}}$ of $+28^{\circ} \mathrm{C}$ compared with an analogous duplex carrying six MMs (Fig. 2D). Hence, the stability of siRND3 $\left(T_{\mathrm{M}}=\right.$ $57.6^{\circ} \mathrm{C}$ ), a population of siRNAs with eight randomized sites, most closely matched the siRNA carrying two mismatches (siRNA3-2MM) $\left(T_{\mathrm{M}}=58^{\circ} \mathrm{C}\right)$.

Taken together, we concluded therefore that for the most part, singlestranded randomized RNAs of siRND3 hybridized to form their most thermodynamically stable (complementary) duplexes.

\section{SiRNA duplexes carrying degenerate sites produce active RISC populations}

To assess the impact of randomized positions in siRNAs on their silencing potential, dual luciferase assays were performed. We cloned a perfectly complementary target site for the antisense strand (AS) of siRNA3 into a Renilla luciferase reporter plasmid, which also carried a firefly luciferase for normalization purposes. First, we investigated siRNAs containing the mismatched base pairs (siRNA3-2MM, -4MM, -6MM; Fig. 2C). Small 
A

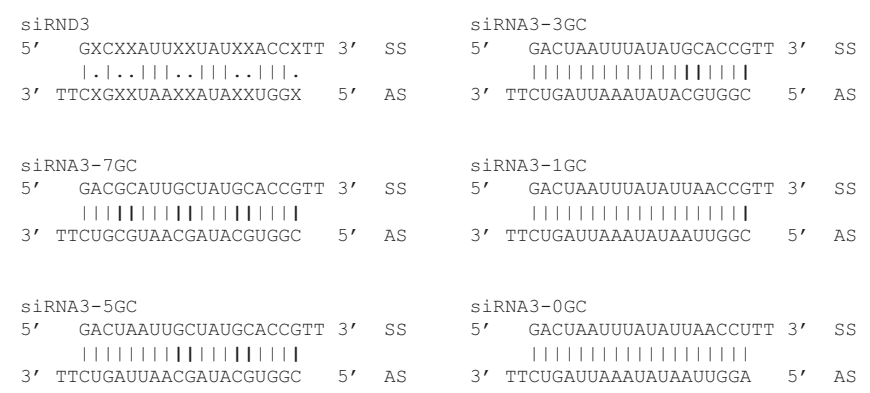

B

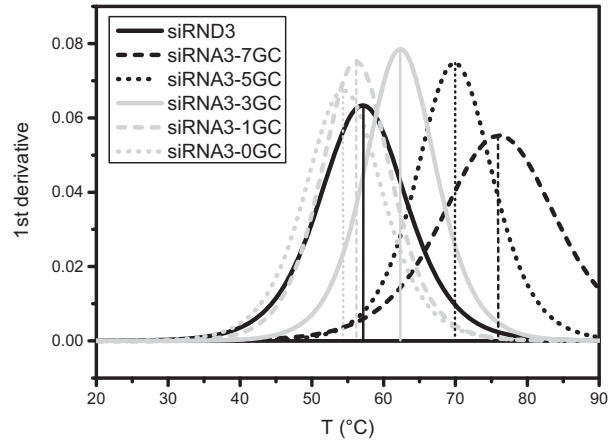

C

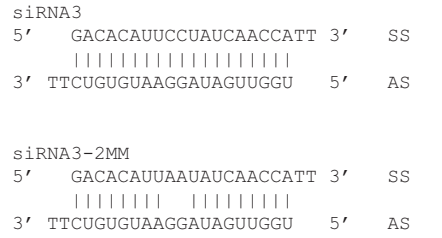

SIRNA3-4MM
5' GACAAAUUAAUAUAAACCATT
$3^{\prime}$ SS

5' GACAAAUUAAUAUAAACCAT
111111111111111

3' titcuguguanggauauuggu $5^{\prime}$ as

SIRNA3-6MM

5' GACCAAUUAAUAUACACCATT $3{ }^{\prime}$ SS

II। II। II। |1।

3' tTCUguguaAggauaguUgG 5 ' AS

D

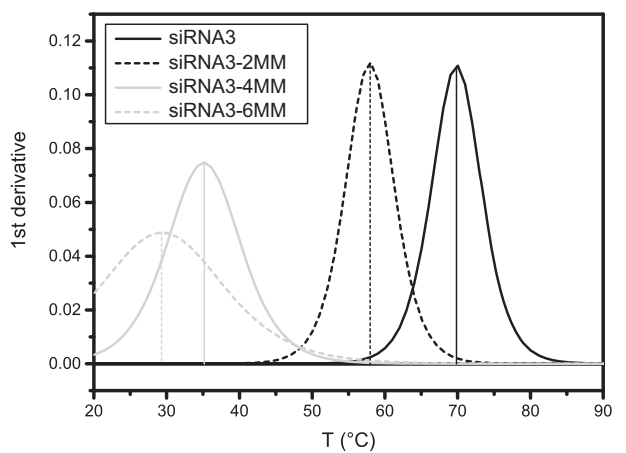

\begin{tabular}{l|l} 
SiRNAs & $T_{M} /{ }^{\circ} \mathrm{C}$ \\
\hline SiRNA3 & $69.9 \pm 0.5$ \\
SiRNA3-2MM & $58.0 \pm 0.5$ \\
SiRNA3-4MM & $35.0 \pm 0.5$ \\
SiRNA3-6MM & $29.4 \pm 0.5$
\end{tabular}

FIGURE 2. Properties of nonrandomized siRNAs. (A) Sequences of siRND3 and nonrandomized siRNA3 control duplexes differing in the number of $\mathrm{G}-\mathrm{C}$ base pairs present $(0-7)$. The sequences of the siRNAs were based on siRND3 and mismatches introduced at the positions where randomizations occurred in siRND3. Watson-Crick base pairs are indicated with a dash. $(B)$ Melting curve analysis of nonrandomized siRNA3 controls. The graph shows the first derivative of melting curves. $T_{\mathrm{M}} \mathrm{s}$ listed in the table represent the mean of triplicate measurements $\pm \mathrm{SD}$. $(C)$ Sequences of the nonrandomized mismatch control siRNA duplexes. The sequences of the siRNAs were based on siRND3 and mismatches introduced at the positions where randomizations occurred in siRND3. Watson-Crick base pairs are indicated with a dash. $(D)$ Melting curve analysis of mismatch controls. The graph shows melting temperatures of nonrandomized siRNA duplexes that carry a defined number of mismatched base pairs. $T_{\mathrm{M}} \mathrm{s}$ listed in the table represent the mean of triplicate measurements \pm SD.

numbers of mismatches in RNA duplexes do not preclude loading into RISC, as evidenced by the loading of natural miRNAs and artificial mismatched siRNAs (Yoda et al. 2010). The siRNAs were cotransfected with the reporter plasmid into HeLa cells (Fig. 3A). We observed a concentration- dependent repression of luciferase by siRNA3 and siRNA3-2MM of eight- to ninefold at the highest concentrations, consistent with a recent report (Yoda et al. 2010) that two centrally placed mismatched base pairs do not attenuate silencing potency. With increasing numbers of mismatches in the sense strand, the repression of luciferase was reduced: 2.2-fold repression for four $\mathrm{MMs}$ (siRNA3-4MM) and 1.5-fold repression for six MMs (siRNA3-6MM) (Fig. 3A). Nevertheless, even with four MMs in the siRNA and a low duplex stability at $37^{\circ} \mathrm{C}$, the guide strand was still able to repress its complementary target. These observations suggested that siRNAs containing up to eight randomized sites, equivalent to approximately two MMs, would also produce RISC-active guide strands. Targeting, however, would be distributed across a large number of individual mRNAs at biologically insignificant levels (Myers et al. 2006). Consistent with this, siRND3 showed weak, nonsignificant repression of luciferase (Fig. 3A).

To support our hypothesis that rndsiRNAs (e.g., siRND3) activate RISC and produce a broadly distributed, low level of silencing, we annealed six sense strands carrying four to eight randomized sites to six antisense strands carrying no randomized sites (Fig. 3B). Randomized positions in the sense strand were base paired to either $\mathrm{G}$ or $\mathrm{U}$, thereby increasing the bona fide base-pairing probability per randomized nucleotide incorporation from $P=0.375$ to $P=0.5$. Two dual luciferase reporter plasmids were then prepared: In one (pTS1-3), target sites for the three antisense strands of AS/RNDs 1-3 were cloned into a single vector; in the second (pTS4-6), target sites for AS/RNDs 4-6 were inserted. The siRNA heteroduplexes were then cotransfected with their appropriate reporter vector into HeLa cells. All siRNA heteroduplexes showed concentrationdependent repression of the target reporter, ranging from 10.8-fold repression in AS/RND4 to 3.5-fold repression in AS/RND6 (Fig. 3C). From these observations, and consistent with the previous experiments, we concluded that the presence of up to eight randomized sites produced on average a sufficiently small number of 
A

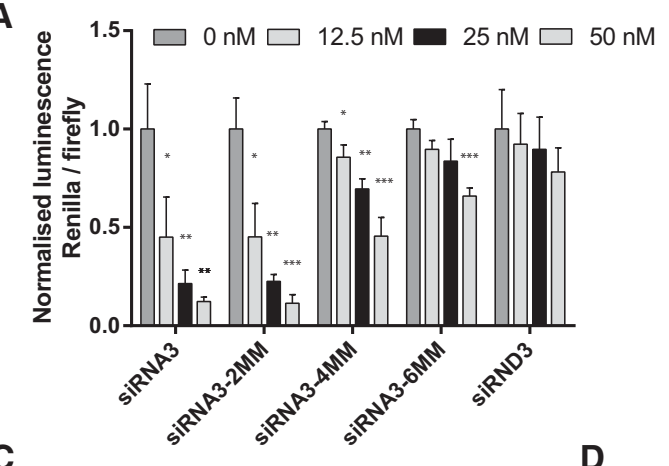

C

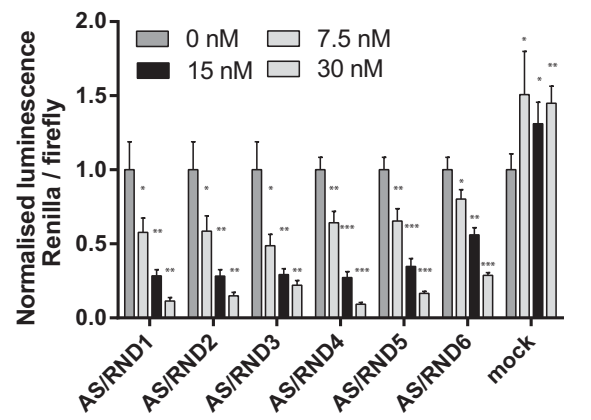

B

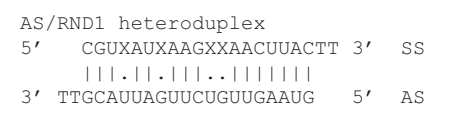

$3^{\prime}$ ttgcaudagudCuguugaAug $5{ }^{\prime}$ AS

AS/RND4 heteroduplex

5' CGCXAUXAAUXXAAUCUACTT $3^{\prime}$ SS

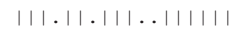

3' tTgcgunaguUauguUagaug 5' AS

AS / RND2 heteroduplex

5' GUGXXUAAXXAACXCAXACTT $3^{\prime}$ SS

|11...111..111.11.11

3' tTcacguauuuguugugugug 5' AS

AS/RND5 heteroduplex

5' GCUAXUXGAAXXAACUUACTT $3^{\prime}$ SS

$1111.1 .111 \ldots .1111111$

3' ttcgaudauduuggudganug 5' As

AS/RND3 heteroduplex

GXCXXAUUXXUAUXXACCXTT

$|.| \ldots|| \ldots|+| \ldots|| \mid$.

3' TTCUGUGUAAGGAUAGUUGGU

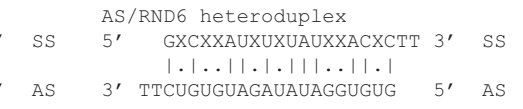

E
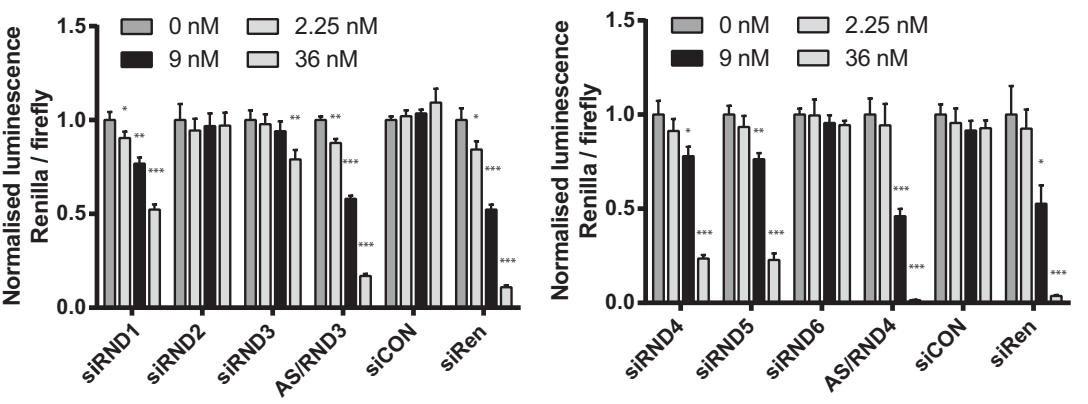

FIGURE 3. Silencing properties of siRNDs. (A) Dual luciferase reporter assay. SiRND3 and the nonrandomized mismatch controls were cotransfected with a dual luciferase reporter plasmid carrying a target site for siRNA3 antisense strand. Relative Renilla/firefly luciferase activity (hRLuc/ hluc+) normalized to the $0-\mathrm{nM}$ dose is shown in means of triplicate transfections \pm SD. (B) Sequences of siRND sense strand and nonrandomized siRNA antisense strand heteroduplexes 1-6. Watson-Crick base pairs are indicated with a dash. Randomized positions are indicated with a dot. ( $C$ ) Dual luciferase reporter assay. AS/RND siRNA heteroduplexes 1-6 were cotransfected with a reporter plasmid carrying the respective nonrandomized target site. Mock treatment corresponded to empty reporter plasmid transfection (psiCHECK-2) with increasing doses of AS/RND4. Relative luciferase activity (hRLuc/hluc+) was normalized to the $0 \mathrm{nM}$ treatment and shown in means of triplicate transfections $\pm \mathrm{SD}$. (D,E) Dual luciferase reporter assays. Rnd-siRNAs were cotransfected with a reporter plasmid carrying the respective target sites. Heteroduplexes AS/RND's 3 and 4 were used as positive controls for the target site. SiRen and siCon served as positive and negative controls for the dual luciferase plasmid. Relative luciferase activity (hRLuc/hluc+) was normalized to the $0 \mathrm{nM}$ treatment and shown in means of triplicate transfections \pm SD.

mismatches in the heteroduplex that RISC loading and target silencing was not dramatically affected.

\section{Position of randomized sites influences the silencing properties of rnd-siRNAs}

Next, we assessed the influence of strand position of randomized sites on silencing. We cotransfected siRNDs 1-3 and siRNDs 4-6 together with reporter plasmids TS1-3 and TS4-6, respectively, into HeLa cells and measured luminescence $72 \mathrm{~h}$ post-transfection. The siRNA heteroduplexes AS/RND3 and AS/RND4 were used as positive controls. We also included previously characterized siRNAs targeting Renilla (siRen) and the negative control siRNA (siCon). The silencing efficiency of siRen on both constructs was similar, whereas siCon was inactive (Fig. 3D,E). SiRND1, siRND4, and siRND5 showed significant silencing of their reporters (1.9-fold, 4.2-fold, and 4.4-fold at the highest concentrations, respectively). SiRND3 was slightly active (1.3-fold). SiRND2 and siRND6 were inactive. Overall, we saw that rnd-siRNAs with randomized sites in the seed region (nt 2-6) of the antisense strand (siRND2, siRND3, siRND6) were largely inactive. In contrast, rnd-siRNAs with no randomized sites in the seed region (siRND1, siRND4, siRND5) showed robust concentration-dependent target suppression (Fig. 3D,E), highly suggestive of a miRNA-like mechanism of inhibition. In fact, siRND1 and siRND5, which bear the same seed region in their antisense strands, showed similar targeting efficiency when normalized to the activity of siRen on pTS1-3 and pTS4-6, respectively $(P=0.1634$; data not shown). Furthermore, comparing the performance of AS/RND4 with that of siRND4 revealed that the former is $\sim 16$-fold more potent, consistent with their presumed siRNA-like and miRNA-like mechanisms, respectively.

In a post-analysis of three genome-wide siRNA screens conducted to uncover genes involved in bacterial/viral infections (Franceschini et al. 2014), investigators concluded that the majority of the phenotypic effects were not derived from the full siRNA sequence but rather from the siRNA seed regions. To assess the effects of an rnd-siRNA with randomized positions in the seed on a whole-transcriptome scale, we transfected HeLa cells with siRND2 at $40 \mathrm{nM}$ and purified total RNA $72 \mathrm{~h}$ post-transfection. Total RNA was used for small RNA sequencing library preparation and the samples 
sequenced on an Illumina HiSeq2000. We included siCon and mock treatments as references. An unrelated siRNA tested in the same experiment served as a control for efficient transfection (data not shown). We compared the siRND2 and siCon treatments to the mock-treated samples. We found that siRND2-treated cells showed a smaller variance of transcript levels when compared with mock cells than siCon-treated cells. This can be seen by the longer "tail" in the distribution of expression fold changes (Fig. 4A). SiRND2 caused a moderate increase in two transcripts but it did not reduce the level of any mRNA with statistical significance $(P$-value $<0.05)$. In contrast, siCon treatment produced overall a longer tail of suppressed mRNAs and in addition, significantly repressed 15 transcripts (Fig. 4A; Supplemental Tables 5, 6). These observations are consistent with the data from the aforementioned reporter assays, suggesting that although siRND2 is a substrate for RISC, it has no inherent miRNA-like or siRNA-like inhibitory activity.

The treatment of cells with dsRNA reagents often have anti-proliferative effects and can even cause apoptosis. We therefore assessed the effects of rnd-siRNA transfections on apoptosis induction using caspase-3/7 luminescent assays. We used miRNA-34a-5p mimic as a positive control, as we have shown previously that this mimic has a pro-apoptotic effect (Guennewig et al. 2014), and siRNA3 and siCon as conventional siRNAs. We transfected HeLa cells with different doses of dsRNAs and measured the conversion of a luminescent caspase-3/7 substrate in the supernatants (Fig. 4B) and lysates (data not shown) of cells to gauge apoptosis induction caused by the treatments. Robust (6.6-fold) induction of apoptosis was found from miR-34a-5p mimic at the 25-nM concentration compared with mock-transfected cells. The nonrandomized siRNA3 as well as siCon induced apoptosis from two- to threefold at the same concentrations. SiRND1 appeared to reduce the caspase-3/7 activity, whereas siRND4 induced apoptosis 1.8 -fold. The seed sequence of siRND 4 (UAGAUU) has no known functional miRNA counterpart; however, it is possible that the sequence UXGC on the AS strand might be responsible for apoptosis induction: When the randomized position in this motif is occupied with a G, a UGGC stretch is present. This motif was previously identified as immunostimulatory (Fedorov et al. 2006) and is not present in any of the other rnd-siRNAs. SiRNA3 may induce apoptosis via a UGUGU motif on the AS strand that has been shown to be immunostimulatory in a previous study (Judge et al. 2005). SiRND2, siRND3, siRND5, and siRND6 showed no significant induction of apoptosis.

\section{Toward better negative controls for RNAi experiments}

The unexpected properties of rnd-siRNAs prompted us to investigate their potential as a novel class of negative control reagents for RNAi experiments. A good negative control for any given effector siRNA has two important properties: It does not exhibit any "sequence-dependent" siRNA- or miRNAlike effects, and it shows the same "sequence-independent" effects as the effector siRNA.

We transfected rnd-siRNAs into HeLa cells at two concentrations and analyzed total RNA $24 \mathrm{~h}$ post-transfection using RT-qPCR (Supplemental Table 4) for a set of genes with wellcharacterized roles in cell cycle regulation, TGF- $\beta$ and IFN- $\gamma$ signaling, immunostimulation, and apoptosis. Target expression levels in rnd-siRNA-transfected cells were compared with mock-treated cells (Fig. 4C). SiCon negative control served as a benchmark. Of the eight genes, CDKN1A expression was stable to all treatments. As expected, many of the genes were affected by the treatments: three genes showed induction $(S P 1, P T E N, C C N D 1)$ and three genes were repressed (RB1, PKR, SMAD2) by rnd-siRNAs as well as siCon, $24 \mathrm{~h}$ post-transfection. Given the diversity of the guide sequences in the six rnd-siRNAs treatments, we concluded that the common effects on the six marker genes were sequence-independent effects of dsRNA treatments in this experiment, which could not be attributed to siRNA- and miRNA-like activities. This conclusion cannot be drawn from analysis of the data for siCon, a conventional chemically unmodified siRNA, which based on previously published findings probably exhibits a combination of sequence-dependent and sequence-independent OTEs. Taken together, we conclude that rnd-siRNAs can be used to reveal genes susceptible to sequence-independent OTEs of effector siRNAs in RNAi experiments.

\section{DISCUSSION}

In this study, we have investigated the preparation and properties of dsRNAs containing randomized base pairs. We synthesized them on solid-phase using an equimolar mixture of RNA phosphoramidites for coupling steps at defined positions. We confirmed by deep sequencing of one RNA that all possible sequences were represented in the library and therefore a very high level of sequence diversity was obtained, but incorporation of randomized bases at some positions was possibly not perfectly randomized. We measured the thermal stability of several rnd-siRNA pools. By comparing their $T_{\mathrm{M}} \mathrm{s}$ with those of analogous complementary and mismatched duplexes, we concluded that in a rnd-siRNA the average number of mismatches at randomized positions was much smaller than expected for a random annealing of randomized guide and randomized passenger strands. The data implied that under annealing conditions two highly diverse RNA populations each with almost $40 \%$ of the sequence randomized were, to a large extent, able to locate and hybridize to complementary partners in a diverse population of RNAs. It is conceivable that with a perfectly random incorporation of nucleotides at degenerate positions during synthesis, one could assemble a population of perfectly complementary siRNAs over time. We then confirmed that rnd-siRNAs duplexes provide guide strands that are loaded into RISC and 

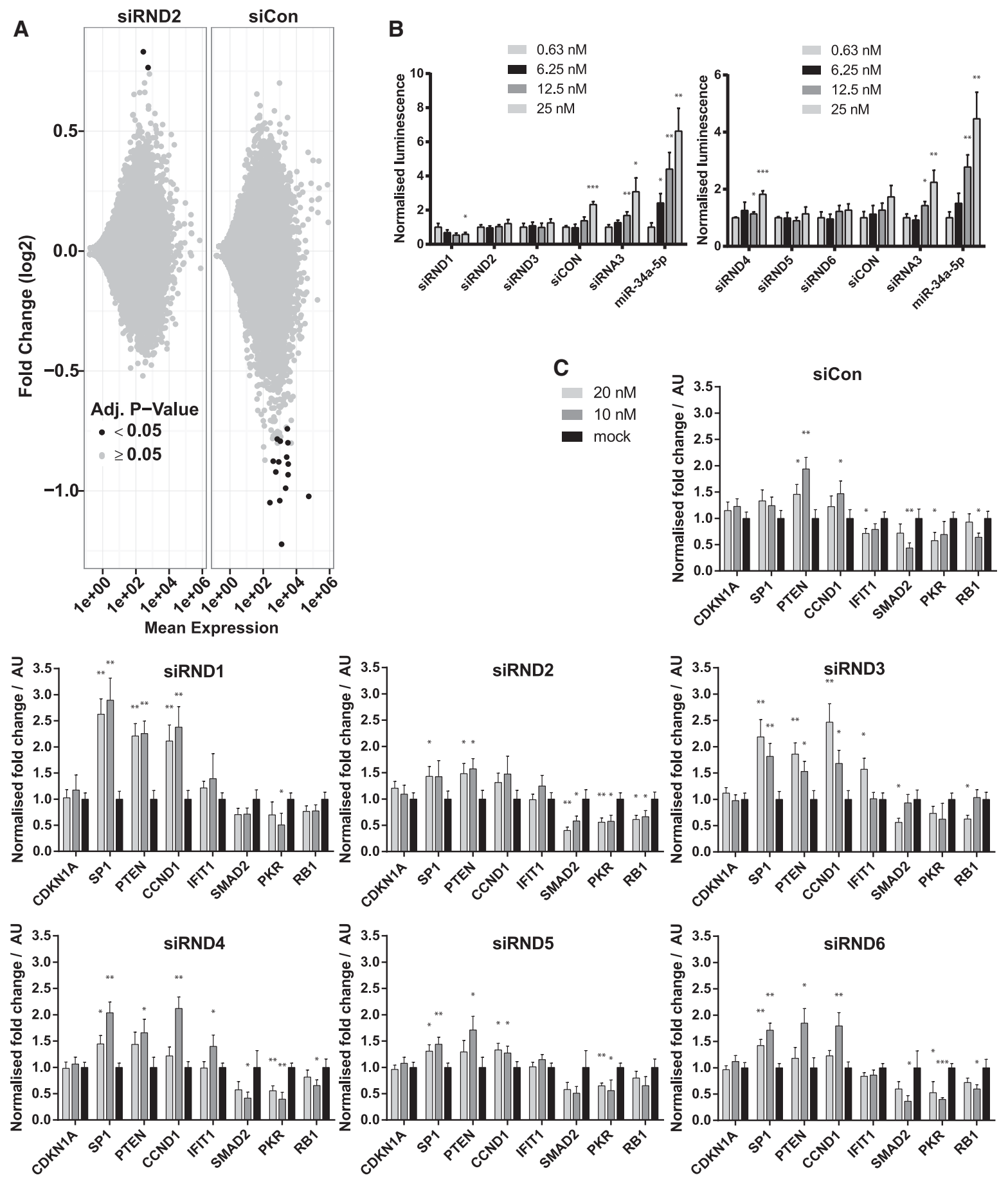

FIGURE 4. Sequence-independent effects of siRNDs. (A) MA plot comparing gene expression of cells from treatments with siRND2 and siCon versus a mock treatment. Differential expression analysis was performed using DESeq2. $P$-values for differential expression were adjusted for multiple testing using the Benjamini-Hochberg procedure and are shown in gray if $<0.05$. (B) Caspase $3 / 7$ assays using supernatants of siRNA transfected HeLa cells. The figure shows caspase- $3 / 7$ activity $72 \mathrm{~h}$ post-transfection. Cells were transfected with siRNDs, siRNA3, or siCon. MicroRNA 34a-5p mimic served as a positive control. Data from averaged triplicates of one representative experiment are shown. Error bars represent \pm SD. (C) SYBR green RT-qPCR analysis of rnd-siRNA transfections. SiRNDs and siCon were transfected into HeLa cells and the expression of select mRNAs involved in cell viability was measured. $C_{\mathrm{p}}$ values were normalized to GAPDH/ACTB and calibrated to the mock transfection. Bars represent the average normalized fold change of triplicate measurements \pm SD from one representative experiment.

are capable of post-transcriptional gene silencing. Interestingly, but consistent with previous findings (Doench et al. 2003), randomized guide strands were able to silence effi- ciently target reporters when their seed regions were devoid of randomized positions, presumably by a miRNA-like mechanism. We found by means of RNA deep sequencing that 
siRND2 treatment caused fewer gene perturbations of overall smaller intensity than a nonrandomized, commercially available control siRNA. Testing of a small panel of genes associated with cell signaling, immunostimulation, and apoptosis using qPCR provided insight that rnd-siRNAs induced sequence-independent off-target effects similar to nonrandomized siRNAs.

There has been much discussion on how to best design and use negative controls in RNAi experiments (Whither RNAi? [Editorial] 2003). A good negative control sequence for an effector siRNA is one that helps to confirm that the phenotypic effect produced by the effector siRNA is due to the inhibition of its intended target. Here, we suggest that the unique properties of rnd-siRNAs render them a new pragmatic class of negative control siRNAs. They are superior to conventional controls (scrambled, commercially available nontargeting controls, mismatch controls) because they will not silence the effector's target, they do not exhibit their own sequence-dependent effects, and thus they can reveal sequence-independent effects in an RNAi experiment. Compared with other classes of pooled siRNAs that might be used as negative controls (e.g., esiRNAs or diced siRNAs), they hold additional advantages: They are easy to chemically synthesize reproducibly and quickly in large quantities, and they have a massive sequence complexity, yet they can be designed to avoid inclusion of known toxic motifs (e.g., UGGC; Fedorov et al. 2006). For large-scale screening applications or experiments using multiple siRNAs, an rnd-siRNA such as siRND2 can be of value as a general negative control. Testing these reagents and applications in our laboratory is under further investigation.

\section{MATERIALS AND METHODS}

\section{Cell culture and transfections}

HeLa cells (ATCC, CCL-2) were maintained in Dulbecco's Modified Eagle's medium (Gibco, Invitrogen) supplemented with $10 \%$ fetal bovine serum (FBS; Sigma-Aldrich). SiCon negative control siRNA (Silencer Negative Control No. 5 AM4642) was purchased from Ambion. The miRIDIAN microRNA mimic hsa-miR-34a-5p (MIMAT0000255) was purchased from Dharmacon. Renilla siRNA was obtained from Dharmacon (5'-GAGCGAAGAGGGCGAGAAA UU-3'). RNAs were transfected into HeLa cells using oligofectamine (No. 12252-011, Invitrogen) according to the manufacturer's instructions. Plasmid vectors were transfected into HeLa cells using jetPEI (Polyplus).

\section{Cloning of target sites into dual luciferase reporter plasmid}

DNA oligonucleotides (sense and antisense) containing tandem target sites complementary to either siRND's $1-3$ or siRND's $4-6$ were chemically synthesized (Supplemental Table 2), annealed and cloned into psiCHECK-2 (Promega) dual reporter plasmids (pTS1-3, pTS4-6) using XhoI and NotI restriction endonucleases. Ligated plasmids were transformed and propagated in DH5alpha competent cells. Incorporation of the inserts was validated by sequencing of the extracted plasmids.

\section{Randomized siRNA design}

Six different siRNA randomized duplexes were designed with the following properties: 21-nt RNA strands each with 29\%-50\% GC content, $\mathrm{G}$ or $\mathrm{C}$ at the $5^{\prime}$ end of each strand, $2 \mathrm{nt}$ (dTdT) overhangs at the $3^{\prime}$ ends. The siRNA ends were designed to have similar G/C content and stability taking into account the eight last base pairs of the duplex. Next we introduced four, six, or eight randomized bases per strand in such a way that randomized positions would pair in the annealed siRNA. The probability of randomized pairs to form a Watson-Crick or G-U base pair, assuming a perfect randomized mixture of all 4-nt bases, is $P_{(x=1)}=0.375$. For eight randomizations the total amount of mismatched bases would account to $5\left(8 \times\left(1-P_{(x=1)}\right)=8 \times 0.625=5\right)$. The sequences were designed with maximally two consecutive randomized positions per strand in order to minimize the size of internal loops (Supplemental Table 1). SiRND4 and siRND5 were based on the randomization pattern of siRND1, with siRND5 carrying the same hexamer seed sequence as siRND1 in the antisense strand. SiRNDs 1, 4, and 5 carried intact hexamer seeds that were not present in any known miRNA. Sense and antisense strands of rnd-siRNAs showed no consensual alignment to reference sequence RNA using nucleotide BLASTn algorithm (search parameters: word size $=7$, Match/Mismatch score $=$ $1,-3$, Gap cost $=5,2)$. As strand bias in siRNA loading is thought to be evoked by differences in the thermodynamic stability of the siRNA ends (Khvorova et al. 2003; Schwarz et al. 2003), we tried to place similar numbers of randomized bases at both duplex ends. Randomized positions have a mismatch probability of $62.5 \%$ and therefore reduce the duplex stability. A similar thermodynamic stability of the duplex ends should evenly distribute loading of both strands into RISC. SiRNDs 1, 4, and 5 showed asymmetrical end stability, carrying two randomizations at the $5^{\prime}$ sense strand duplex end, whereas the other duplex end carried none in the last $7 \mathrm{nt}$ (sense strand should be loaded preferentially on to RISC in those siRNAs). Additionally, we analyzed the predicted silencing potency of sense and antisense strands individually using a set of 17 nonredundant criteria defined by four publications (Amarzguioui and Prydz 2004; Reynolds et al. 2004; Ui-Tei et al. 2004; Pei and Tuschl 2006). Based on those parameters the two strands were predicted to be similarly potent in most siRNDs, with the exception of siRND4, where we found a potency bias for the antisense strand (Supplemental Table 3). We calculated the number of individual siRNA strand sequences contained per rnd-siRND (permutations), assuming a perfect randomization $\left(P_{b}=0.25\right)$ (Supplemental Table 1). We found that a minimum amount of 7.15 fmol of RNA was required per rnd-siRNA in order to obtain one duplex of every possible siRNA combination for $x=8$ randomizations:

$$
n=\frac{b^{x} \times b^{x}}{N_{\mathrm{A}}}
$$

where $n=$ RNA in moles, $N_{\mathrm{A}}=$ Avogadro constant, $b=$ four RNA bases (A, G, C, U), $x=$ randomized positions per strands, assuming that all siRNA strands would form duplexes. The amount of siRND synthesized ranged from 20 to $40 \mathrm{nmol}$ thereby assuring that the full combinatory potential of our approach was tapped (at least $2.8 \times 10^{6}$ unique strand molecules). The lowest possible transfection dose 
(7.15 fmol) in $80 \mu \mathrm{L}$ volume would equal a minimal dose of 89.4 $\mathrm{pM}$, which was not undershot in this study.

\section{Randomized RNA synthesis}

The rnd-siRNAs were synthesized on a MerMade 12 synthesizer (Bioautomation Corporation) in DMT-On mode. We used phosphoramidites (Thermo Fisher Scientific) and UnySupport controlled-pore glass $500 \AA$ solid support (CPG; Glen Research). Randomized positions were synthesized using an equimolar mixture of all four phosphoramidites (A, G, C, U) neglecting a possible sequence bias due to different coupling efficiencies of the individual RNA building blocks. Oligonucleotides were deprotected using gaseous methylamine at $70^{\circ} \mathrm{C}$ and $1.2 \mathrm{bar}$ for $2 \mathrm{~h}$. RNAs were vacuumdried at $25^{\circ} \mathrm{C}$ and eluted with ethanol/water (1:1). The deprotection of the $2^{\prime}$ TBDMS group was carried out by addition of a premixed solution of dry 1-methyl-2-pyrrolidone, triethylamine and triethylamine-trihydrofluoride $(6: 3: 4 \mathrm{ratio})$ for $90 \mathrm{~min}$ at $70^{\circ} \mathrm{C}$. Ethoxytrimethylsilane was added to quench the reaction and RNA was lyophilized at $25^{\circ} \mathrm{C}$. RNA pellets were dissolved in doubly deionized water $\left(\mathrm{ddH}_{2} \mathrm{O}\right)$ and purified using reverse-phase high performance liquid chromatography (RP-HPLC, Agilent 1200 Series; Agilent Technologies). The collected oligonucleotide was deprotected using $40 \%$ acetic acid for $60 \mathrm{~min}$ at $25^{\circ} \mathrm{C}$. The dried pellet was again dissolved in $\mathrm{ddH}_{2} \mathrm{O}$ and purified by HPLC using a $\mathrm{C} 18$ column (XBridge OST, particle size $2.5 \mathrm{~mm}$, Waters). Purified samples were analyzed on an Agilent 6130 Series Quadrupole LC/MS (Agilent Technologies) with electron spray ionization. Purity and yields were determined by HPLC and Nanodrop 2000 (Thermo Scientific) measurements, respectively. RNA was stored in aqueous solution at $-20^{\circ} \mathrm{C}$.

\section{RNA deep sequencing of siRND3 antisense strand}

SiRND3 antisense strand linked to a $5^{\prime}$ RNA adapter (Illumina TruSeq RA5) was synthesized (5'GUUCAGAGUUCUACAGUCCG ACGAUCXGGUXXAUAXXAAUXXGXC-dTdT3'). The purified oligonucleotide was ligated to a $3^{\prime}$ adapter (Illumina TruSeq RA3) using T4 RNA ligase2 (1-249, K227Q, NEB). Ligation products were PCR-amplified using DreamTaq polymerase (Thermo Scientific) and standard RP index primers (Illumina). Amplification products were purified and sequenced on an Illumina HiSeq2000. Sequencing data were demultiplexed and analyzed using the Galaxy web server (https://usegalaxy.org/). Sequencing reads were clipped and low quality reads (Phred $<13$ ) and reads $<21 \mathrm{nt}$ removed. Data matching the complete 21 -nt-long siRND-AS sequence were selected (.GGT..ATA..AAT..G.CTT pattern) and a consensus motif created using WebLogo3 from a total of 3.3 million reads. Raw data are accessible online (http://www.genomespace.org/) with file name: "BSSE_QGF_20638_CAGATC_random_siRND3AS.fastq.gz".

\section{RNA sequencing and differential expression analysis}

RNA was extracted from cells using TRIzol/PCA and poly $(A)^{+}$RNAs enriched using Dynabeads Oligo(dT)25 kit (Life Technologies). Subsequently, RNA was fragmented by adding $50 \mu \mathrm{L}$ of alkaline buffer ( $50 \mathrm{mM} \mathrm{NaCO}_{3}, 1 \mathrm{mM}$ EDTA, pH 9.2) and heating $5 \mathrm{~min}$ at $95^{\circ} \mathrm{C}$ followed by cooling on ice. Purified RNAs were dephosphor- ylated using fastAP enzyme (Life Technologies) and rephosphorylated using polynucleotide kinase (T4 PNK, NEB) after purification. Next a preadenylated $3^{\prime}$ sequencing adapter (Illumina TruSeq RA3, 5'-rAppTGGAATTCTCGGGTGCCAAGG-3' ordered from IDT) was ligated using T4 RNA Ligase 2, truncated K227Q (NEB). Subsequently, the $5^{\prime}$ RNA adapter (Illumina TruSeq RA5, 5'-GUUCAGAGUUCUACAGUCCGACGAUC- $3^{\prime}$ ) was ligated with T4 RNA ligase (NEB), and samples were reverse transcribed using Superscript III RT (Life Tech.) and RT primer (RTP). PCR pilot reactions were carried out using DreamTaq polymerase (Fermentas) with universal RP-1 forward primer and RPI-n indexed reverse primers. After identification of the optimal cycle numbers a final PCR was carried out as described before. The PCR products were purified using Agencourt AMPure XP kit (Beckman-Coulter) and a small aliquot checked on analytical $2 \%$ agarose TBE gels. If the quality was satisfactory, cDNA libraries were sent to the Biozentrum Basel where samples were sequenced using an Illumina HiSeq2000. RNA-Seq reads in FASTQ format were trimmed using Trimmomatic 0.30 (Bolger et al. 2014) and aligned to Genome Reference Consortium Human genome build 38 (GRCh38) using STAR 2.4.0h (Dobin et al. 2013). The read counts for ENSEMBL 78 protein coding genes were quantified using HTSeq-count (Anders et al. 2015), discarding multimapping reads, as recommended by the authors for differential expression analysis. Differential expression analysis was performed using the Bioconductor package DESeq2 (Love et al. 2014). Read counts were normalized using the median ratio method (Anders and Huber 2010) and dispersion for each gene was estimated using the methods of Cox Reid-adjusted likelihood maximization (McCarthy et al. 2012). Negative binomial generalized linear models (GLMs) were fitted and tested for significance of coefficients using the Wald test in two scenarios: siCon vs. Mock and siRND2 vs. Mock, each time testing for significantly differentially expressed genes through the addition of a specific negative control siRNA against the control experiment without transfection. $P$-values for differential expression were adjusted for multiple testing using the Benjamini-Hochberg procedure.

\section{Polyacrylamide gel electrophoresis (PAGE)}

Native $10 \%$ polyacrylamide gels with $0.75 \mathrm{~mm}$ thickness were prepared. After $30 \mathrm{~min}$ of polymerization, gels were prerun in a Mini-Protean Tetra Cell (BioRad) electrophoresis chamber for 20 min at 80 V. $50 \mathrm{ng}$ of RNA duplexes or $100 \mathrm{ng}$ of single-stranded RNAs were prepared in a nondenaturing loading dye $(2.5 \%$ Ficoll 400, $1 \mathrm{mM}$ EDTA, $0.025 \%$ bromophenol blue) and loaded. The gel was run for $30 \mathrm{~min}$ at $80 \mathrm{~V}$ followed by $45 \mathrm{~min}$ at $100 \mathrm{~V}$ or until the bromophenol blue band was approaching the end of the gel. The gel was stained using GelRed (Biotium) and an image was acquired using ChemiDoc XRS (BioRad) light cabinet.

\section{Melting curve acquisition}

Melting temperatures of RNA duplexes were measured on a CARY 300 (Agilent) equipped with a thermocontroller. SiRNA sense and antisense strands were combined to yield an equimolar concentration of $2 \mu \mathrm{M}$ in phosphate-buffered saline $\left(2.5 \mathrm{mM} \mathrm{Na}_{2} \mathrm{HPO}_{4}\right.$, $5 \mathrm{mM} \mathrm{NaH}_{2} \mathrm{PO}_{4}, 100 \mathrm{mM} \mathrm{NaCl}, 0.5 \mathrm{mM}$ EDTA). Absorptions at $260 \mathrm{~nm}$ were measured in $80 \mu \mathrm{L}$ quartz cuvettes. The temperature gradient was set to $0.1 \times K \times \mathrm{min}^{-1}(0.5 \mathrm{~K} / \mathrm{min}$ for Fig. 2D) for 
the range of $20^{\circ} \mathrm{C}-90^{\circ} \mathrm{C}$. Absorbance readings were taken every 30 sec. Each series was performed three times. Hold time was set to 5 min at $90^{\circ} \mathrm{C}$ and $20^{\circ} \mathrm{C}$, respectively, to ensure thermal equilibrium. The melting profiles were fitted to a sigmoidal and $T_{\mathrm{M}}$ was determined by the derivative thereof.

\section{Caspase-3/7 luminescent assays}

Cell viability of RNA-transfected HeLa cells was analyzed using a chemiluminescent substrate (Caspase-Glo 3/7 assay system, Promega). Apoptosis induction was measured $72 \mathrm{~h}$ post-transfection in supernatants or cell lysates. Luminescent caspase substrate $(5 \mu \mathrm{L})$ was added to $5 \mu \mathrm{L}$ of supernatant in a 384 -well white microtitre plate. After 30-min incubation at room temperature, luminescence was measured on a Mithras LB940 luminometer (Berthold Technologies).

\section{Dual luciferase reporter assays}

Complementary target sites for either nonrandomized siRNA 1, 2, and 3 AS strands or nonrandomized siRNA 4, 5, and 6 AS strands (Fig. 3B) were cloned into psiCHECK-2 Vector (Promega). HeLa cells were seeded in 96-well white plates at a density of 7000 cells per well and RNAs were transfected in triplicates after at least 5-h incubation with oligofectamine (Life Technologies). After $24 \mathrm{~h}$, plasmid DNA (40 ng/well) was transfected using jetPEI (Polyplus) according to the manufacturer's protocol. After $72 \mathrm{~h}$, supernatants were removed and firefly substrate $(15 \mu \mathrm{L}$ including Lysis Buffer; Dual-Glo Luciferase Assay System, Promega) diluted with $15 \mu \mathrm{L}$ $\mathrm{H}_{2} \mathrm{O}$ was added. Luminescence was measured on a luminometer (Mithras LB940, Berthold Technologies). After $30 \mathrm{~min}, 15 \mu \mathrm{L}$ of Renilla substrate (including firefly Quencher Solution; Dual-Glo Luciferase Assay System, Promega) per well was added and the measurement was repeated. Values were normalized to firefly luciferase at the $0 \mathrm{nM}$ treatment.

\section{RNA extraction and RT-qPCR}

Total RNA was extracted from HeLa cells $24 \mathrm{~h}$ post-transfection using a TRIzol/PCA protocol (Rio et al. 2011) and quality and yield assessed using Nanodrop 2000. Total RNA ( $1 \mu \mathrm{g}$ ) was reverse transcribed using $\mathrm{ABI}$ high capacity cDNA reverse transcription kit (4368814, Life Technologies) according to the manufacturer's instructions. Expression levels were assayed using Roche SYBR Green PCR Master Mix (Roche). The quantitative real-time polymerase chain reaction was carried out on a LightCycler 480 (Roche) using the following cycling conditions: $10 \mathrm{~min}$ at $95^{\circ} \mathrm{C}$ (activation), 40 cycles $\left(15 \mathrm{sec}\right.$ at $95^{\circ} \mathrm{C} ; 60 \mathrm{sec}$ at $60^{\circ} \mathrm{C}$ ) followed by the acquisition of a melting curve from $95^{\circ} \mathrm{C}-40^{\circ} \mathrm{C}$ (ramp rate $0.11^{\circ} \mathrm{C} / \mathrm{sec}, 5$ acquisitions $/ \mathrm{sec}$ ). $C_{\mathrm{p}}$ values and melting curves were extracted using LightCycler Software V1.5 (Roche) and analyzed using the $2^{\Delta \Delta C_{p}}$ method. GAPDH and $A C T B$ mRNA were used for normalization and mock cells as calibrators. Mock cells were treated with oligofectamine equivalent to the $10 \mathrm{nM}$ treatment. Primer sequences can be found in the supplement (Supplemental Table 3). Standard deviation for relative fold changes was calculated using error propagation (Nordgård et al. 2006).

\section{SUPPLEMENTAL MATERIAL}

Supplemental material is available for this article.

\section{ACKNOWLEDGMENTS}

We thank M. Zimmermann for help with RNA synthesis and transfections and Jochen Imig for reagents and help with the deep sequencing experiment. This work was supported in part by grants from the Swiss National Science Foundation to J.H. (205321_124720 and 205320_144123).

Received August 1, 2015; accepted September 11, 2015.

\section{REFERENCES}

Aleman LM, Doench J, Sharp PA. 2007. Comparison of siRNA-induced off-target RNA and protein effects. RNA 13: 385-395.

Amarzguioui M, Prydz H. 2004. An algorithm for selection of functional siRNA sequences. Biochem Biophys Res Commun 316: 1050-1058.

Anders S, Huber W. 2010. Differential expression analysis for sequence count data. Genome Biol 11: R106.

Anders S, Pyl PT, Huber W. 2015. HTSeq-a Python framework to work with high-throughput sequencing data. Bioinformatics 31: 166-169.

Birmingham A, Anderson EM, Reynolds A, Ilsley-Tyree D, Leake D, Fedorov Y, Baskerville S, Maksimova E, Robinson K, Karpilow J, et al. 2006. 3' UTR seed matches, but not overall identity, are associated with RNAi off-targets. Nat Methods 3: 199-204.

Bolger AM, Lohse M, Usadel B. 2014. Trimmomatic: a flexible trimmer for Illumina sequence data. Bioinformatics 30: 2114-2120.

Buchholz F, Kittler R, Slabicki M, Theis M. 2006. Enzymatically prepared RNAi libraries. Nat Methods 3: 696-700.

Buehler E, Chen YC, Martin S. 2012. C911: A bench-level control for sequence specific siRNA off-target effects. PLoS One 7: e51942.

Clark PR, Pober JS, Kluger MS. 2008. Knockdown of TNFR1 by the sense strand of an ICAM-1 siRNA: dissection of an off-target effect. Nucleic Acids Res 36: 1081-1097.

Dobin A, Davis CA, Schlesinger F, Drenkow J, Zaleski C, Jha S, Batut P, Chaisson M, Gingeras TR. 2013. STAR: ultrafast universal RNA-seq aligner. Bioinformatics 29: 15-21.

Doench JG, Petersen CP, Sharp PA. 2003. siRNAs can function as miRNAs. Genes Dev 17: 438-442.

Echeverri CJ, Beachy PA, Baum B, Boutros M, Buchholz F, Chanda SK, Downward J, Ellenberg J, Fraser AG, Hacohen N. 2006. Minimizing the risk of reporting false positives in large-scale RNAi screens. Nat Methods 3: 777-779.

Elbashir SM, Harborth J, Lendeckel W, Yalcin A, Weber K, Tuschl T. 2001. Duplexes of 21-nucleotide RNAs mediate RNA interference in cultured mammalian cells. Nature 411: 494-498.

Fedorov Y, Anderson EM, Birmingham A, Reynolds A, Karpilow J, Robinson K, Leake D, Marshall WS, Khvorova A. 2006. Off-target effects by siRNA can induce toxic phenotype. RNA 12: 1188-1196.

Franceschini A, Meier R, Casanova A, Kreibich S, Daga N, Andritschke D, Dilling S, Ramo P, Emmenlauer M, Kaufmann A, et al. 2014. Specific inhibition of diverse pathogens in human cells by synthetic microRNA-like oligonucleotides inferred from RNAi screens. Proc Natl Acad Sci 111: 4548-4553.

Ghildiyal M, Zamore PD. 2009. Small silencing RNAs: an expanding universe. Nat Rev Genet 10: 94-108.

Guennewig B, Roos M, Dogar AM, Gebert LF, Zagalak JA, Vongrad V, Metzner KJ, Hall J. 2014. Synthetic pre-microRNAs reveal dualstrand activity of miR-34a on TNF- $\alpha$. RNA 20: 61-75.

Hannus M, Beitzinger M, Engelmann JC, Weickert MT, Spang R, Hannus S, Meister G. 2014. siPools: highly complex but accurately defined siRNA pools eliminate off-target effects. Nucleic Acids Res 42: 8049-8061. 
Hornung V, Guenthner-Biller M, Bourquin C, Ablasser A, Schlee M, Uematsu S, Noronha A, Manoharan M, Akira S, de Fougerolles A, et al. 2005. Sequence-specific potent induction of IFN- $\alpha$ by short interfering RNA in plasmacytoid dendritic cells through TLR7. Nat Med 11: 263-270.

Jackson AL, Linsley PS. 2010. Recognizing and avoiding siRNA off-target effects for target identification and therapeutic application. Nat Rev Drug Discov 9: 57-67.

Jackson AL, Burchard J, Schelter J, Chau BN, Cleary M, Lim L, Linsley PS. 2006. Widespread siRNA "off-target" transcript silencing mediated by seed region sequence complementarity. RNA 12: $1179-1187$.

Judge AD, Sood V, Shaw JR, Fang D, McClintock K, MacLachlan I. 2005. Sequence-dependent stimulation of the mammalian innate immune response by synthetic siRNA. Nat Biotechnol 23: 457-462.

Khvorova A, Reynolds A, Jayasena SD. 2003. Functional siRNAs and miRNAs exhibit strand bias. Cell 115: 209-216.

Leuschner PJ, Ameres SL, Kueng S, Martinez J. 2006. Cleavage of the siRNA passenger strand during RISC assembly in human cells. EMBO Rep 7: 314-320.

Love MI, Huber W, Anders S. 2014. Moderated estimation of fold change and dispersion for RNA-seq data with DESeq2. Genome Biol 15: 550 .

Mandecki W. 1990. A method for construction of long randomized open reading frames and polypeptides. Protein Eng 3: 221-226.

Marine S, Bahl A, Ferrer M, Buehler E. 2012. Common seed analysis to identify off-target effects in siRNA screens. J Biomol Screen 17: 370-378.

Matranga C, Tomari Y, Shin C, Bartel DP, Zamore PD. 2005. Passengerstrand cleavage facilitates assembly of siRNA into Ago2-containing RNAi enzyme complexes. Cell 123: 607-620.

McCarthy DJ, Chen Y, Smyth GK. 2012. Differential expression analysis of multifactor RNA-Seq experiments with respect to biological variation. Nucleic Acids Res 40: 4288-4297.

Myers JW, Chi JT, Gong D, Schaner ME, Brown PO, Ferrell JE. 2006. Minimizing off-target effects by using diced siRNAs for RNA interference. J RNAi Gene Silencing 2: 181-194.

Nordgård O, Kvaløy JT, Farmen RK, Heikkilä R. 2006. Error propagation in relative real-time reverse transcription polymerase chain reaction quantification models: the balance between accuracy and precision. Anal Biochem 356: 182-193.

Parsons BD, Schindler A, Evans DH, Foley E. 2009. A direct phenotypic comparison of siRNA pools and multiple individual duplexes in a functional assay. PLoS One 4: e8471.
Pei Y, Tuschl T. 2006. On the art of identifying effective and specific siRNAs. Nat Methods 3: 670-676.

Rand TA, Petersen S, Du F, Wang X. 2005. Argonaute2 cleaves the anti-guide strand of siRNA during RISC activation. Cell 123: 621-629.

Reynolds A, Leake D, Boese Q, Scaringe S, Marshall WS, Khvorova A. 2004. Rational siRNA design for RNA interference. Nat Biotechnol 22: 326-330.

Rio DC, Ares M, Hannon GJ, Nilsen TW. 2011. RNA: a laboratory manual. Cold Spring Harbor Laboratory Press, Cold Spring Harbor, NY.

Saxena S, Jonsson ZO, Dutta A. 2003. Small RNAs with imperfect match to endogenous mRNA repress translation. Implications for off-target activity of small inhibitory RNA in mammalian cells. J Biol Chem 278: 44312-44319.

Schwarz DS, Hutvágner G, Du T, Xu Z, Aronin N, Zamore PD. 2003. Asymmetry in the assembly of the RNAi enzyme complex. Cell 115: 199-208.

Shin C, Nam JW, Farh KK, Chiang HR, Shkumatava A, Bartel DP. 2010. Expanding the microRNA targeting code: functional sites with centered pairing. Mol Cell 38: 789-802.

Sledz CA, Holko M, de Veer MJ, Silverman RH, Williams BR. 2003. Activation of the interferon system by short-interfering RNAs. Nat Cell Biol 5: 834-839.

Ui-Tei K, Naito Y, Takahashi F, Haraguchi T, Ohki-Hamazaki H, Juni A, Ueda R, Saigo K. 2004. Guidelines for the selection of highly effective siRNA sequences for mammalian and chick RNA interference. Nucleic Acids Res 32: 936-948.

Vickers TA, Lima WF, Wu H, Nichols JG, Linsley PS, Crooke ST. 2009. Off-target and a portion of target-specific siRNA mediated mRNA degradation is Ago2 'Slicer' independent and can be mediated by Ago1. Nucleic Acids Res 37: 6927-6941.

Wada A, Yabuki S, Husimi Y. 1980. Fine structure in the thermal denaturation of DNA: high temperature-resolution spectrophotometric studies. CRC Crit Rev Biochem 9: 87-144.

Whither RNAi? [Editorial] 2003. Nat Cell Biol 5: 489-490.

Yoda M, Kawamata T, Paroo Z, Ye X, Iwasaki S, Liu Q, Tomari Y. 2010. ATP-dependent human RISC assembly pathways. Nat Struct Mol Biol 17: 17-23.

Zon G, Gallo KA, Samson CJ, Shao KL, Summers MF, Byrd RA. 1985. Analytical studies of 'mixed sequence' oligodeoxyribonucleotides synthesized by competitive coupling of either methyl- or $\beta$-cyanoethyl- $N, N$-diisopropylamino phosphoramidite reagents, including 2'-deoxyinosine. Nucleic Acids Res 13: 8181-8196. 

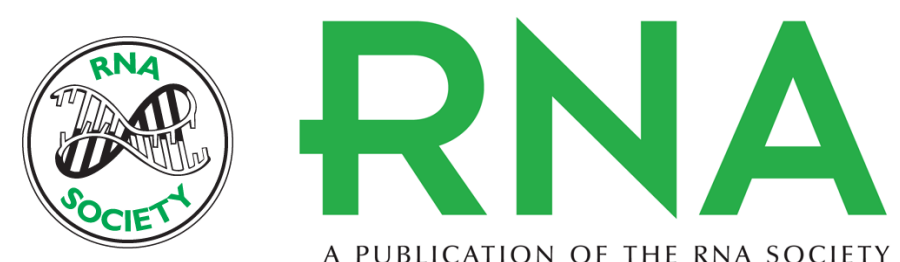

A PUBLICATION OF THE RNA SOCIETY

\section{Properties of short double-stranded RNAs carrying randomized base pairs: toward better controls for RNAi experiments}

Julian A. Zagalak, Mirjam Menzi, Fabian Schmich, et al.

RNA 2015 21: 2132-2142 originally published online October 29, 2015

Access the most recent version at doi:10.1261/rna.053637.115

\section{Supplemental http://rnajournal.cshlp.org/content/suppl/2015/10/08/rna.053637.115.DC1 Material}

References This article cites 45 articles, 8 of which can be accessed free at: http://rnajournal.cshlp.org/content/21/12/2132.full.html\#ref-list-1

Creative This article is distributed exclusively by the RNA Society for the first 12 months after the Commons License full-issue publication date (see http://rnajournal.cshlp.org/site/misc/terms.xhtml). After 12 months, it is available under a Creative Commons License (Attribution-NonCommercial 4.0 International), as described at http://creativecommons.org/licenses/by-nc/4.0/.
Email Alerting Receive free email alerts when new articles cite this article - sign up in the box at the Service top right corner of the article or click here.

\section{III!" PI Providing Precise Solutions for your research.}

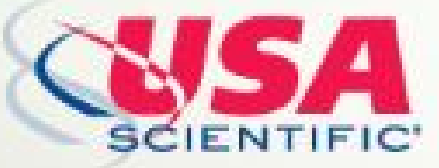

To subscribe to $R N A$ go to:

http://rnajournal.cshlp.org/subscriptions

(C) 2015 Zagalak et al.; Published by Cold Spring Harbor Laboratory Press for the RNA Society 